\title{
Functioning thyroglossal cyst and concurrent ectopic thyroid in the floor of the mouth without an orthotopic thyroid gland
}

Shruti Dhingra ${ }^{1}$, MS, DNB, Achal Gulati ${ }^{1}$, MS, Abhishek $\underline{\text { Bansal }}^{2}$, MD

\begin{abstract}
We report the case of a 20 -year-old man with a rare constellation of anomalies - a functional thyroglossal cyst and concurrent ectopic thyroid in the floor of the mouth, without an orthotopic thyroid gland. To our knowledge, this is the third such report in the otolaryngology literature. It is important to be aware of the possibility of concurrent ectopic thyroid and thyroglossal cyst in the absence of an orthotopic thyroid gland. The majority of reported cases of thyroid ectopy occur at the base of the tongue. The occurrence of an ectopic thyroid in the floor of the mouth is both unusual and not amenable to clinical examination. Even if there is good uptake on nuclear imaging, the ectopic thyroid may be hypofunctioning. We discuss the importance of thyroid imaging, as well as the need for preoperative imaging and an appropriate management strategy in the treatment of patients with this triad of anomalies.
\end{abstract}

Keywords: congenital anomalies, ectopic thyroid, thyroglossal cyst

\section{INTRODUCTION}

Thyroglossal duct cyst is one of the most common anomalies encountered during the embryological development of the thyroid gland. It typically presents as a midline neck mass in the region of the hyoid bone. Ectopic thyroid tissue is known to occur anywhere in the pathway of descent of the thyroid gland. Although cases of ectopic thyroid in the floor of the oral cavity have been reported, its occurrence is rare. These anomalies may exist without a functioning orthotopic thyroid gland. However, the coexistence of these anomalies is rare, with only two cases previously reported in the literature. ${ }^{(1,2)}$ In this report, we describe the coexistence of a thyroglossal cyst (with functioning thyroid tissue) and an ectopic thyroid in the floor of the oral cavity, without an orthotopic thyroid gland.

\section{CASE REPORT}

A 20-year-old Indian man presented to the otolaryngology clinic with a mass in the anterior neck. The mass had been present for the past ten years, but had recently increased in size. The patient had no history of dysphagia, dyspnoea or voice change, and denied any radiation exposure or familial thyroid disease. Clinical examination revealed a mass, measuring approximately $2.0 \mathrm{~cm} \times 1.5 \mathrm{~cm}$, in the midline of the anterior neck. The mass had a smooth surface and a soft consistency, and moved when the patient swallowed. Fineneedle aspiration cytology revealed abundant colloid, many follicular cells, few lymphocytes and clusters of columnar cells. Ultrasonography of the neck confirmed the presence of a heterogeneous mass with multiple small, nodular components and a cystic area suggestive of a thyroglossal

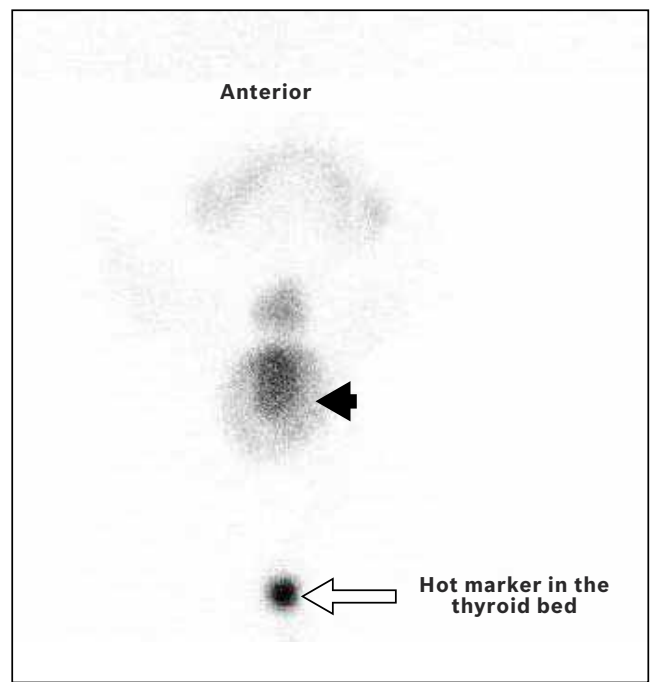

Fig. 1 Technetium-99m pertechnetate image shows a well-defined focus (arrowhead) of tracer concentration in the region of the clinically palpable swelling. Note the diffuse tracer uptake immediately above the well-defined focus, which corresponds to the mass in the floor of the mouth, and the lack of uptake in the site of the normal thyroid gland.

cyst with thyroid tissue remnants. Technetium-99m pertechnetate imaging showed (a) a well-defined focus of tracer concentration in the region of the clinically palpable swelling, with diffuse tracer uptake immediately above the well-defined focus, corresponding to the mass in the floor of the mouth; and (b) no uptake at the site of the normal thyroid gland (Fig. 1). No soft tissue mass or bulge was identified at the base of the tongue on fibreoptic examination. The patient's thyroid profile showed a normal total triiodothyronine (T3) level of $121.6 \mathrm{ng} / \mathrm{dL}$ (normal 86-186 ng/dL), a total thyroxine (T4) level of $6.9 \mu \mathrm{g} \mathrm{dL}$

\footnotetext{
${ }^{1}$ Department of Otolaryngology and Head and Neck Surgery, Maulana Azad Medical College and Lok Nayak Jai Prakash Narayan Hospital, ${ }^{2}$ Department of Radiological Imaging, Institute of Nuclear Medicine and Allied Sciences, New Delhi, India

Correspondence: Dr Shruti Dhingra, Senior Resident, Department of Otolaryngology and Head and Neck Surgery, Maulana Azad Medical College and Lok Nayak Jai Prakash Narayan Hospital, New Delhi 110002, India. shrudoc@hotmail.com
} 

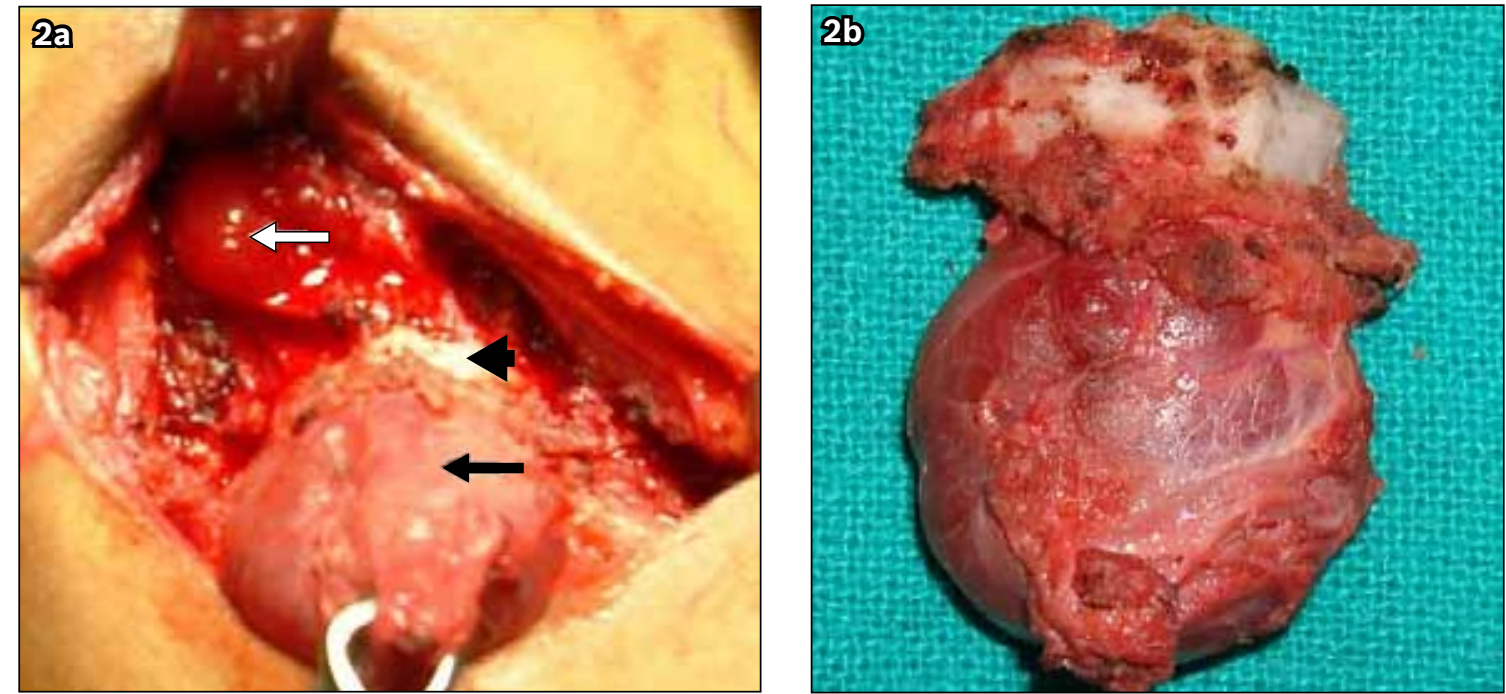

Fig. 2 (a) Intraoperative photograph shows the thyroglossal cyst (black arrow) with the hyoid bone. A smooth, pinkish mass (white arrow) was observed just above the hyoid bone (arrowhead) in the floor of the oral cavity. (b) Photograph shows the excised thyroglossal cyst and body of hyoid bone.

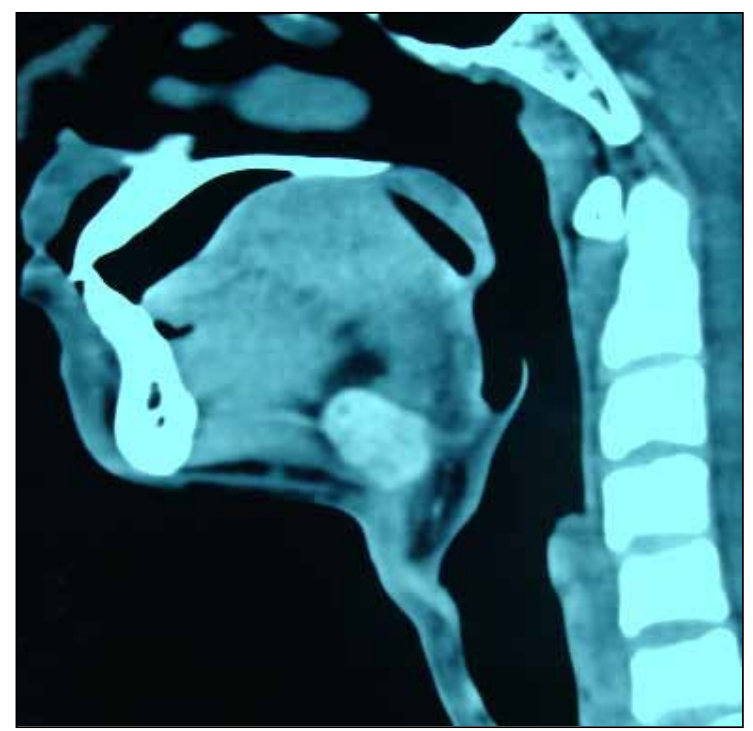

Fig. 3 Postoperative, contrast-enhanced, sagittal CT image shows a homogenously enhancing mass in the posterior floor of the mouth.

(normal 4.5-12.5 $\mu \mathrm{g} / \mathrm{dL}$ ), and a moderately elevated thyroid-stimulating hormone (TSH) level of $10.4 \mu \mathrm{IU} / \mathrm{mL}$ (normal $0.25-4.5 \mu \mathrm{lU} / \mathrm{mL}$ ), which is indicative of a subclinical hypothyroid state. As the patient was previously not on any thyroid hormone treatment, he was started on medical therapy for his hypothyroid status.

As the patient desired surgical removal of the neck mass for cosmetic and psychological reasons, excision using Sistrunk's procedure was agreed upon. However, a decision was made to preserve the ectopic thyroid in the floor of the mouth, as it did not cause any significant symptoms and its preservation would reduce the patient's exogenous thyroid intake. Under general anaesthesia, the patient's anterior neck mass was excised en bloc along with the body of the hyoid bone via a transverse cervical incision. Intraoperatively, another smooth, pinkish mass was observed just above the body of the hyoid, in the region of the floor of the mouth and above the fibres of the mylohyoid muscle (Fig. 2). Since an orthotopic thyroid was absent in the patient, the ectopic thyroid tissue was left in place to prevent severe hypothyroidism postoperatively. Histological examination of the thyroglossal cyst revealed the presence of thyroid tissue with nodular hyperplasia and cystic components with chronic inflammatory cells.

The postoperative period was uneventful, and computed tomography imaging confirmed the presence of the ectopic thyroid mass (measuring $1.8 \mathrm{~cm} \times 2.2 \mathrm{~cm} \times 1.6 \mathrm{~cm}$ ) in the floor of the mouth and absence of the thyroid gland in the pretracheal region (Fig. 3). At the six-month postoperative follow-up, the patient was asymptomatic and euthyroid. Subsequently, he was counselled on how to monitor his thyroid status.

\section{DISCUSSION}

The thyroid gland is the first endocrine gland to develop in the human embryo. The anterior two-thirds of the tongue develops from the tuberculum impar, while the posterior one-third develops from the hypobranchial eminence. The junction between these two regions forms the future foramen caecum. The ultimobranchial bodies, one from each side of the neck, are derived from the fourth pharyngeal pouch, and contribute to the formation of parafollicular cells of the thyroid. In the fifth embryonic week, the thyroglossal duct starts to break down. If remnants of the duct persist, they may form a thyroglossal duct cyst or an ectopic thyroid anywhere along the pathway of migration from the foramen caecum to the mediastinum, with or without an orthotopic thyroid gland. Although thyroglossal duct remnants usually remain asymptomatic, recurrent infections or suspicion of malignancy may be indications for surgical excision. Preoperative assessment via ultrasonography or thyroid imaging is essential to ensure that the ectopic thyroid is not the only functioning thyroid tissue. 
Lingual thyroid is the most commonly seen thyroid ectopy, accounting for $90 \%$ of reported cases. ${ }^{(1)}$ Although rare, other sites of thyroid ectopy, such as the sublingual region, suprahyoid region, mediastinum, lung, porta hepatis, duodenum, oesophagus, heart, breast and intratracheal region, have also been described. ${ }^{(2)}$ However, ectopic thyroid tissue may not function like normal thyroid tissue even though it may show good uptake on nuclear imaging. Ectopic thyroid may also exist in isolation and be the only functioning thyroid tissue, or it may coexist with a functioning orthotopic thyroid gland.(3) A lingual thyroid may remain asymptomatic, but an increase in its size may result in dysphagia, a change in voice or respiratory difficulty, especially during puberty, pregnancy or in times of emotional stress. A lingual thyroid can be visualised on fibreoptic examination if it is located at the base of the tongue. Notably, ectopic thyroid tissue in the sublingual or suprahyoid region may remain clinically asymptomatic for a prolonged duration and is not amenable to clinical examination. The coexistence of a thyroglossal duct cyst and an ectopic thyroid in the floor of the mouth, in the absence of an orthotopic thyroid gland, is extremely rare. Only two cases of this triad of anomalies have been previously reported..$^{(4,5)}$

In most cases, the use of ultrasonography to confirm the presence of the orthotopic thyroid gland in the neck is sufficient to plan for surgical excision of the thyroglossal duct cyst. Technetium-99m pertechnetate imaging, which picks up activity in ectopic sites, is used to determine whether the orthotopic thyroid is the only functioning thyroid in the body. Radiological investigations such as magnetic resonance (MR) imaging may be considered in rare instances when uptake is observed in unusual sites (e.g. the floor of the mouth) where ultrasonography may be inaccurate or difficult to perform. A useful diagnostic tool, MR imaging allows for comparison of postoperative changes in the size of the gland. However, treatment of ectopic thyroid is typically unnecessary unless its size increases considerably causing dyspnoea, dysphagia or bleeding. If hypothyroidism is present, hormone replacement therapy should result in the involution of the ectopic thyroid, especially in cases where excision of the thyroglossal cyst is indicated in the absence of an orthotopic thyroid gland. Patients should be counselled on potential lifelong dependence on exogenous levothyroxine, as the ectopic thyroid may or may not be functionally sufficient. ${ }^{(6)}$

To our knowledge, this is the third report of the coexistence of a thyroglossal cyst with functioning thyroid tissue and an ectopic thyroid in the floor of the oral cavity, without an orthotopic thyroid gland in the neck. Endocrine assessment and nuclear imaging are essential in identifying the location of the functioning thyroid gland, especially if it is present at sites not amenable to clinical examination. As the ectopic thyroid gland may be functionally insufficient, the need for surgical excision should be carefully assessed. If surgical excision is performed, postoperative follow-up is necessary.

\section{REFERENCES}

1. Choi JY, Kim JH. A case of an ectopic thyroid gland at the lateral neck masquerading as a metastatic papillary thyroid carcinoma. J Korean Med Sci 2008; 23:548-50.

2. Batsakis JG, El-Naggar AK, Luna MA. Thyroid gland ectopias. Ann Otol Rhinol Laryngol 1996; 105:996-1000.

3. Mace AT, McLaughlin I, Gibson IW, Clark LJ. Benign ectopic submandibular thyroid with a normotopic multinodular goitre. J Laryngol Otol 2003; 117:739-40.

4. McCoul ED, de Vries EJ. Concurrent lingual thyroid and undescended thyroglossal duct thyroid without orthotopic thyroid gland. Laryngoscope 2009; 119:1937-40.

5. Manohar K, Bhattacharya A, Kashyap R, Kamaleshwaran KK, Mittal BR. Concurrent sublingual thyroid and thyroglossal cyst with functioning thyroid tissue in the absence of an orthotopic thyroid gland. Jpn J Radiol 2010; 28:552-4.

6. Rovet JF. Children with congenital hypothyroidism and their siblings: do they really differ? Pediatrics 2005; 115:e52-7. 